\section{Rhabdomyosarcoma mimicking lymphangioma: report of three cases}

\author{
Silvana Guerriero, ${ }^{1}$ Lorenza Ciracì, ${ }^{1}$ \\ Ermete Giancipoli, ${ }^{1}$ Maria Grazia Fiore, ${ }^{2}$ \\ Domenico Piscitelli² \\ 'Department of Ophthalmology; \\ 2Department of Pathology, University \\ of Bari, Italy
}

\begin{abstract}
We report three cases of proptosis, in children aged 6, 10 and 12, whereby in all cases the first clinical, radiologic and ultrasonographic diagnosis was lymphangioma, while the final anatomopathological diagnosis was rhabdomyosarcoma. In presence of a rapidly worsening exophthalmos or eyelid swelling in a child, an early correct diagnosis is very important. Imaging techniques play a very important role in the diagnosis, but are often inconclusive and an excisional biopsy (if feasible) must always be considered.
\end{abstract}

\section{Introduction}

In presence of a rapidly worsening exophthalmos or eyelid swelling in a child, an early correct diagnosis is very important. Differential diagnosis must include many orbital pathologies: inflammatory lesions such as orbital cellulitis, idiopathic inflammatory orbital pseudotumor, conjunctivitis, and allergic edema, and tumors such as orbital capillary hemangioma, lymphangioma, neuroblastoma, Langerhan's cell histiocytosis and rhabdomyosarcoma. The severity of these diseases is different, as well as the therapeutic intervention and course of the disease Imaging techniques like computed tomography (CT), magnetic resonance imaging (MRI) and ultrasonography play a very important role in the diagnosis, but are often inconclusive.

Between March 2007 and April 2008, three pediatric patients were admitted to our unit. In all three cases imaging findings suggested the diagnosis of a lymphangioma, but only an excisional biopsy led to a definitive diagnosis of rhabdomyosarcoma in all cases.

\section{Case Reports}

\section{Case \#1}

An otherwise healthy 6-year-old Caucasian boy was referred to our unit for surgical removal of a presumed chalazion in the inferior right eyelid (Figure 1A).

Due to rapid worsening of the eyelid swelling, we performed B-scan ultrasonography, which revealed a solid, well-defined mass with a medium-low internal reflectivity, low ultrasound attenuation and signs of internal vascularization (Figure 1B).

CT of the orbit showed a solid mass in the medial-inferior part of the orbit, that appeared homogeneous and hyperdense after contrast enhancement. The lesion infiltrated the orbital soft tissues but spared the orbital bones (Figure 1C and 1D). A new diagnosis of orbital lymphangioma was suggested by radiologist.

The patient underwent trans-eyelid excision of the mass (Figure 2) under general anesthesia. The pathologic result was a solid variant of embryonal rhabdomyosarcoma; striated muscle fibers were evident between sheets of poorly differentiated round tumoral cells that showed hyperchromic nuclei and scarce cytoplasm (Figure 3).

Chemotherapy with cyclophosphamide, actinomycin D and vincristine sulfate and successive radiotherapy ( $40 \mathrm{~Gy}$ ) were instituted. The child continued to demonstrate complete tumor regression at two years follow-up. He developed cataract as a late effect of the radiotherapy, and was successfully operated. His final visual acuity was 20/20.

\section{Case \#2}

An otherwise healthy 10-year-old Caucasian boy was referred to our unit with a one month history of exophthalmos in his left eye (Figure 4A).

Orbital B-scan ultrasonography revealed a solid extraconic neoformation involving the medial segment of the left orbit, $2 \mathrm{~cm}$ across and $3.5 \mathrm{~cm}$ long, well-defined against the surrounding tissues, with a hyporeflective, dyshomogeneous internal reflectivity owing to the presence of septa (Figure 4B).

Orbital CT scans revealed a well-defined lesion with clear margins in the medial segment of the left orbit, causing lateral displacement of the optic nerve and eye. The lesion showed several chambers, which were more evident after contrast enhancement (Figure 4C). T2-weighted MRI images disclosed irregular isointense tissue with an isointense peripheral rim and some central areas with hyperintense signal. On T1-weighted gadolinium-enhanced images the central area of the mass was hypointense. A peripheral hyperintense rim was evident, as well as thin septi dividing the central cystic area, but no fluid levels were present (Figure 4D) These findings were suggestive of lymphangioma containing proteinaceus fluid or hemorrhage.
Correspondence: Silvana Guerriero, Università degli Studi di Bari, Piazza G. Cesare, 11, 70124 Bari, Italy.

Tel. +39.080 .5478 .7916 - Fax: +39.080 .5478 .918 .

E-mail: silvanaguerriero@gmail.com

Key words: rhabdomyosarcoma, lymphangioma, magnetic resonance imaging, computed tomography, sonography.

Conflict of interest: the authors declare no potential conflicts of interests.

Received for publication: 9 April 2011.

Revision received: 21 December 2011.

Accepted for publication: 22 December 2011.

This work is licensed under a Creative Commons Attribution NonCommercial 3.0 License (CC BYNC 3.0).

(C) Copyright S. Guerriero et al., 2012

Licensee PAGEPress, Italy

Eye Reports 2012; 2:e1

doi:10.4081/eye.2012.e1

Since these findings were suggestive of lymphangioma containing proteinaceous fluid, watchful waiting seemed advisable but 15 days later, worsening of the proptosis made it necessary to perform an excisional biopsy (Figure 5).

We performed an anterior trans-eyelid excisional biopsy of the mass under general anesthesia. Intraoperatively, a multicystic encapsulated pink mass with a whitish internal fluid was found. Near-total excision of the mass was performed (Figure 5). Total excision of the mass was not possible because the mass extended posteriorly toward the orbital apex. These findings continued to suggest the diagnosis of a lymphangioma. However, the pathologic result was a solid alveolar rhabdomyosarcoma. Histology showed sheets of poorly differentiated round tumoral cells with hyperchromic nuclei and scarce cytoplasm. Several atypical mitoses were present (Figure 6).

Chemotherapy with cyclophosphamide, actinomycin D and vincristine sulfate and successive radiotherapy (40 Gy) were instituted. The child continued to demonstrate complete tumor regression at two years follow-up. He developed cataract as a late effect of the radiotherapy. His visual acuity is now 10/20.

\section{Case \#3}

An otherwise healthy 12-year-old Caucasian boy was referred to our unit with a 20-day history of eyelid edema (Figure 7A).

We performed B-scan ultrasonography, which revealed a poorly-defined extra-conic solid mass located in the anterior upper segment of the right orbit, $3.0 \mathrm{~cm}$ across, with minor alterations at ultrasound, and a medi- 
um-low internal reflectivity (Figure 7B).

CT scans demonstrated a lobular non-encapsulated mass, featuring the same density as the extra-ocular muscles, well-defined against the surrounding soft tissues, with diffuse contrast enhancement (Figure 7C and 7D).

We performed an anterior trans-eyelid excisional biopsy of the mass under general anesthesia (Figure 8). Histology showed a cell population consisting of small, round tumor cells with hyperchromatic nuclei and of large, polygonal-shaped tumor cells with abundant eosinophilic cytoplasm, which often contained cross striations, alternating with areas of clubshaped tumor cells arranged in clumps and outlined by fibrous septa. In the center, the clusters were arranged loosely, and therefore, they appear in an alveolar pattern. These cells stained intensely with eosinophilic stain. Cross-striated malignant rhabdomyoblasts were observed. The pathologic result was mixed embryonal and alveolar rhabdomyosarcoma (Figure 9).

Chemotherapy with cyclophosphamide, actinomycin D and vincristine sulfate and successive radiotherapy (40 Gy) was instituted. The child continued to demonstrate complete tumor regression at two years follow-up. He developed cataract as a late effect of the radiotherapy, and his visual acuity is now 10/20.

In all three cases the subsequent histopathologic examination disclosed undifferentiated small round cells with hyperchromatic nuclei, a high nuclear-to-cytoplasmic ratio, and a brisk mitotic activity. Immunohistochemistry demon- strated intense immunoreactivity to smooth muscle actin and desmin. These findings led to a definitive diagnosis of rhabdomyosarcoma in all three cases: a solid alveolar rhabdomyosarcoma in the first case, an embryonal rhabdomyosarcoma in the second, and a mixed embryonal and alveolar rhabdomyosarcoma in the last case.

\section{Discussion}

In the presence of exophthalmos during examination in a child, the examiner must be extremely careful to differentiate between various pathologies with a different severity: inflammatory lesions such as orbital cellulitis, idiopathic inflammatory orbital pseudotumor, conjunctivitis, and allergic edema, and tumors such as orbital capillary hemangioma, lymphangioma, neuroblastoma, Langerhan's cell histiocytosis and rhabdomyosarcoma. Most of these conditions can be differentiated by clinical history taking and examination, but orbital lymphangioma may prove to be more challenging to differentiate from rhabdomyosarcoma.

Important elements of differential diagnosis to consider during the orbital exam are: uni or bilateral exophthalmos, the type of progression, slow or rapid, the reducibility, and whether there is pulsatility. The orbital edge must be palpated to look for a mass; the presence of a palpebral redness or thickness orientates towards an orbital cellulitis or a rhab-

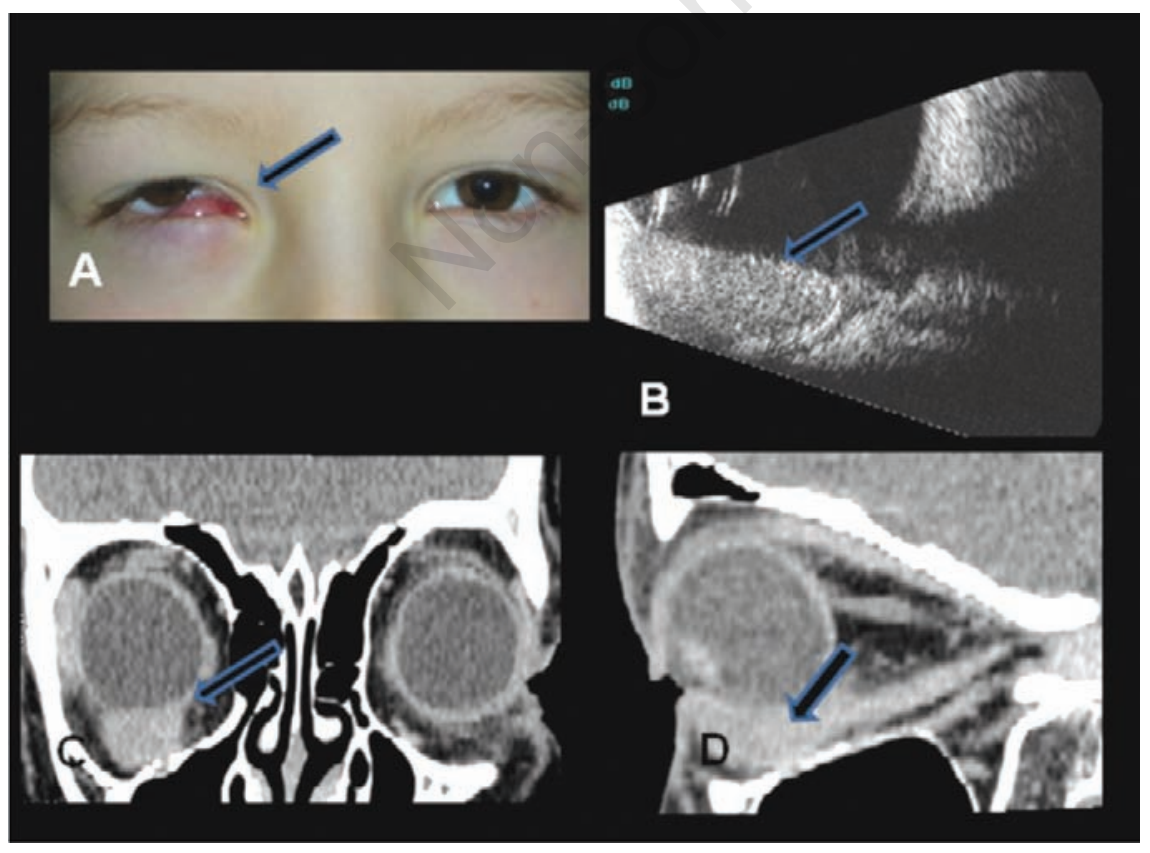

Figure 1. A) Swelling of the right inferior eyelid (arrow). B) B-scan ultrasonography revealed a solid, well defined mass, with low-medium internal reflectivity (arrow). C) and D) CT scans showed a solid mass in the medial-inferior part of the orbit (arrow). domyosarcoma, modification of the eyelid colour when the child cries orientates towards a capillary haemangioma. A rapidly worsening bilateral exophthalmus, if associated with periorbital ecchymosis, is suggestive of a metastatic neuroblastoma, the most common malignant tumor in a child under the age of five. An orbital mass may be a metastasis of a primitive tumor (10\% of cases) located in the retroperitoneal space or in the mediastinum or else a primitive adrenal tumor. A history of orbital pain or headache is more suggestive of an orbital cellulitis. The fundus oculi must be systematically inspected, looking for an optic oedema, optic atrophy, or choroid folds. Other functional restrictions, in particular a reduction of visual acuity and a visual fields deficit, are quite difficult to determine, especially in a child. The physical examination is also very important: coffee and milk spots suggest Recklinghausen's disease. Coloured lesions of the skin could also be present in other diseases, such as histiocytosis and capillary haemangioma. However, in presence of a rapidly worsening and painless exophthalmos, we must always suspect a rhabdomyosarcoma.

Rhabdomyosarcoma is a rare childhood tumor, with an annual incidence of 4.3 cases per million children. ${ }^{1}$ The orbit is the primary site in approximately $10 \%$ of these tumors. ${ }^{2}$ The most frequent clinical findings in patients with ophthalmic rhabdomyosarcoma are proptosis (79\%), globe displacement (79\%), eyelid edema (64\%), and conjunctival congestion (61\%). ${ }^{3}$

Orbital lymphangioma is an uncommon benign cystic lesion generally manifesting in childhood. It accounts for about $1 \%$ of orbital tumors, with no gender preference. ${ }^{3}$ It usually presents with a slowly progressive proptosis, displacement of the globe, ptosis and restriction of eye movements. Occasionally, focal lesions may remain asymptomatic. Spontaneous intraorbital hemorrhage may cause acute proptosis, compressive optic neu-

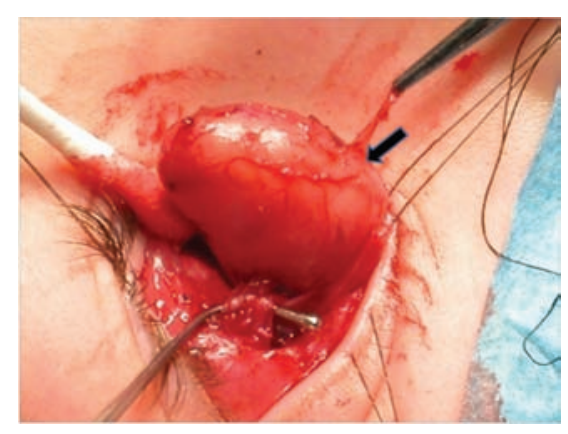

Figure 2. Excisional biopsy (arrow showing the mass). 


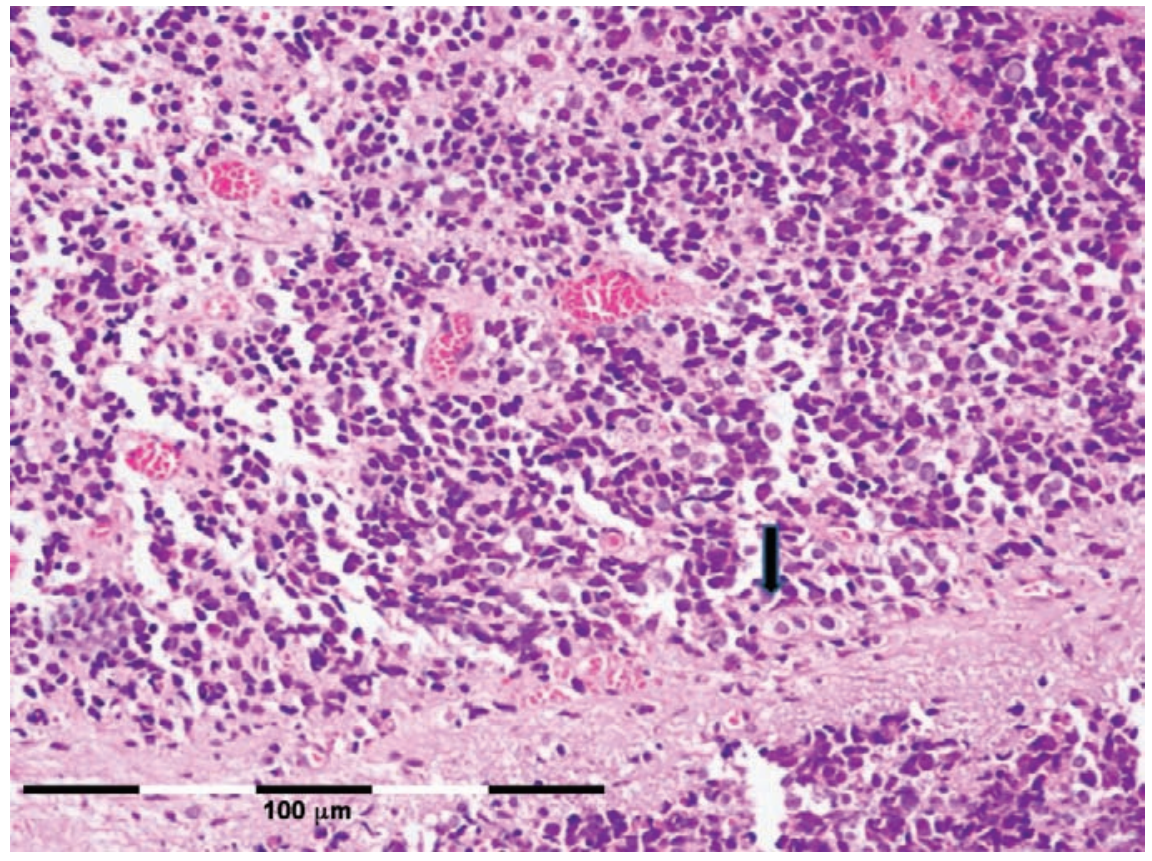

Figure 3. Histology (x100, with hematoxylin and eosin staining, scale bar: $100 \mu \mathrm{m})$ showed a solid variant of embryonal rhabdomyosarcoma; striated muscle fibers (arrow) are evident between sheets of poorly differentiated round tumoral cells, that show hyperchromic nuclei and scarce cytoplasm.

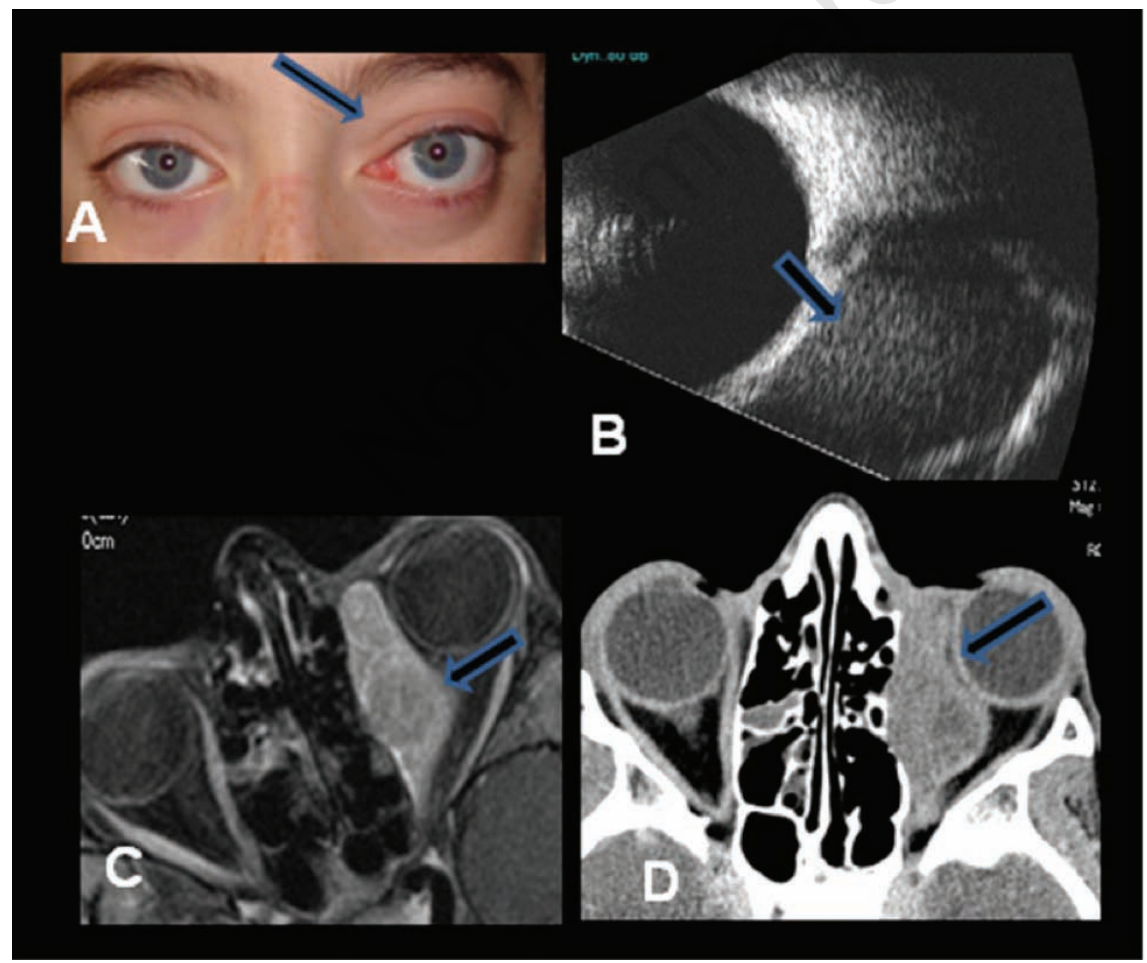

Figure 4. A) Left exophthalmos (arrow). B) B-scan ultrasonography of the mass (arrow). C) CT scans revealed a well-defined lesion with clear margins in the medial segment of the left orbit (arrow). D) MRI revealed a homogeneous aspect of the mass, isointense with the extraocular muscles, and a multitude of internal micro-chambers; a strong enhancement of the lesion was observed after intravenous gadolinium administration (arrow). ropathy and loss of vision. ${ }^{4}$

Rhabdomyosarcoma occurs in patients of the same age group as those with lymphangioma, and both diseases cause painless, noninflammatory proptosis, developing over a short time. Differentiation by orbital imaging is usually helpful and shows a solid enhanced mass with rhabdomyosarcoma versus a multicystic non-enhanced mass with lymphangioma. However, in rare cases, rhabdomyosarcoma can display cavitation, appearing similar to lymphangioma. ${ }^{3}$ The differentiation between rhabdomyosarcoma and lymphangioma seems to be quite challenging because of the frequent overlap of both clinical and radiological aspects of these pathologies. Only the histopathologic examination can confirm the diagnosis. Many other similar cases have been reported in the literature (Table 1): Seedat et al. presented a patient with acute sinusitis whose CT scan showed a ringenhancing lesion within the orbit typical of an orbital subperiosteal abscess. On exploration of the orbit, there was no pus present but a tumour was found, which on histological examination was found to be a rhabdomyosarcoma. ${ }^{5}$ Fetkenhour et al. described a healthy 4year-old girl who presented with an abruptonset proptosis of her right eye with a mild painless swelling of the right upper eyelid during the previous 3 weeks. MRI showed a superonasal heterogeneous soft tissue mass with no bone erosion. On T1-weighted gadoliniumenhanced images, the central area of the mass was hypointense, suggestive of proteinaceous material. A peripheral hyperintense rim indicated vascularized tissue. There was a thin septum dividing the central cystic area, but fluid-fluid levels were not seen. Diagnosis of lymphangioma was favored in light of the rapid development of proptosis, dilated conjunctival lymphatics, subcutaneous ecchymosis, and the presence of presumed cystic, rather than solid, structures on MRI. But histopathologic examination disclosed undifferentiated small round cells with hyperchromatic nuclei, high nuclearto-cytoplasmic ratio, and brisk mitotic activity.

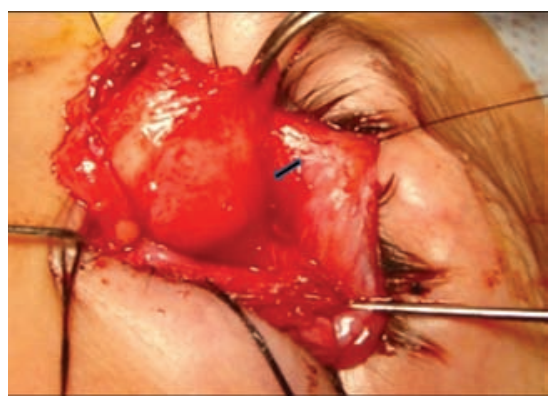

Figure 5. Anterior trans-eyelid excisional biopsy of the mass (the arrow shows the mass). 
Immunohistochemistry demonstrated intense immunoreactivity for smooth muscle actin and desmin. These findings confirmed the diagnosis of rhabdomyosarcoma. ${ }^{6}$ Burkat et al. described a case of a rhabdomyosarcoma mas- querading as heterogeneous soft tissue mass with no bone erosion. On T1-weighted gadolinium-enhanced images, the central area of the mass was hypointense, suggestive of proteinaceous material. A peripheral hyperintense rim

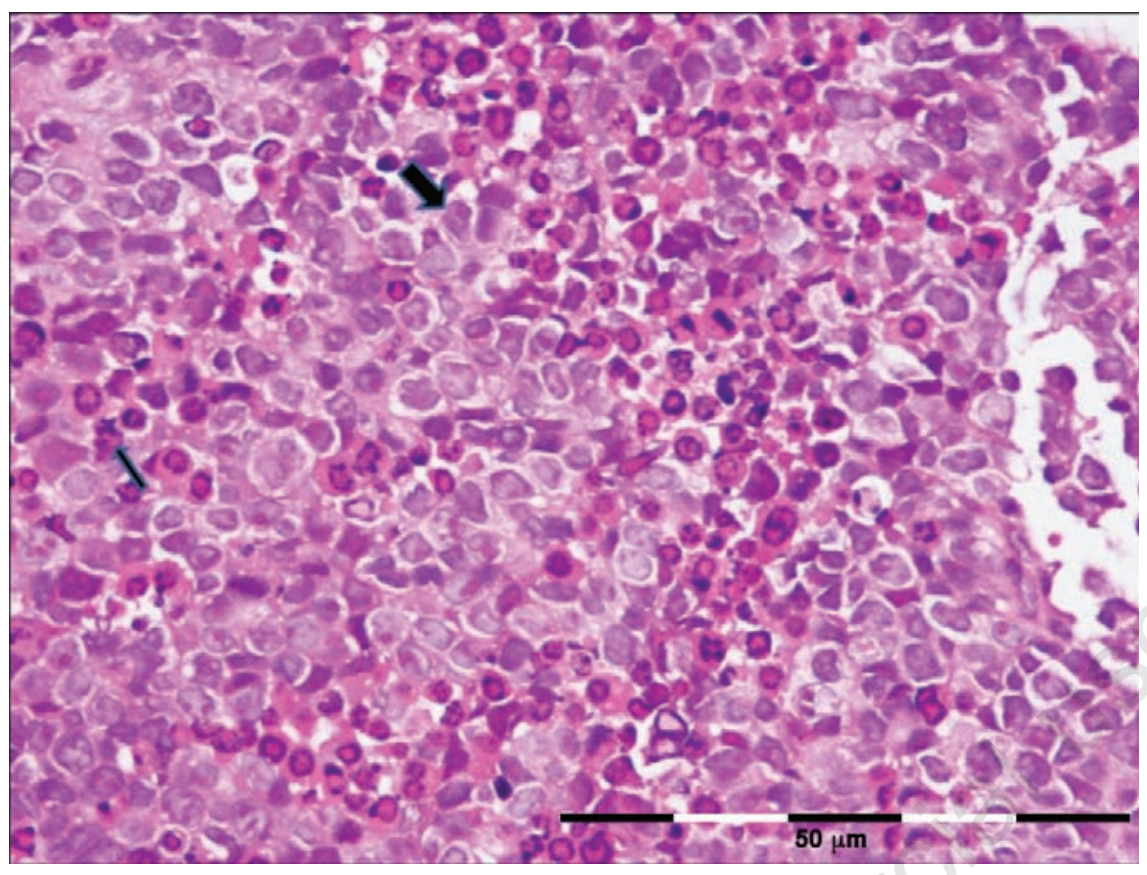

Figure 6. Histology (x400, with hematoxylin and eosin staining, scale bar: $50 \mu \mathrm{m})$ showed a solid variant of alveolar rhabdomyosarcoma, with sheets of poorly differentiated round tumoral cells that show hyperchromic nuclei and scarce cytoplasm (big arrow). Several atypical mitoses are present (small arrow).

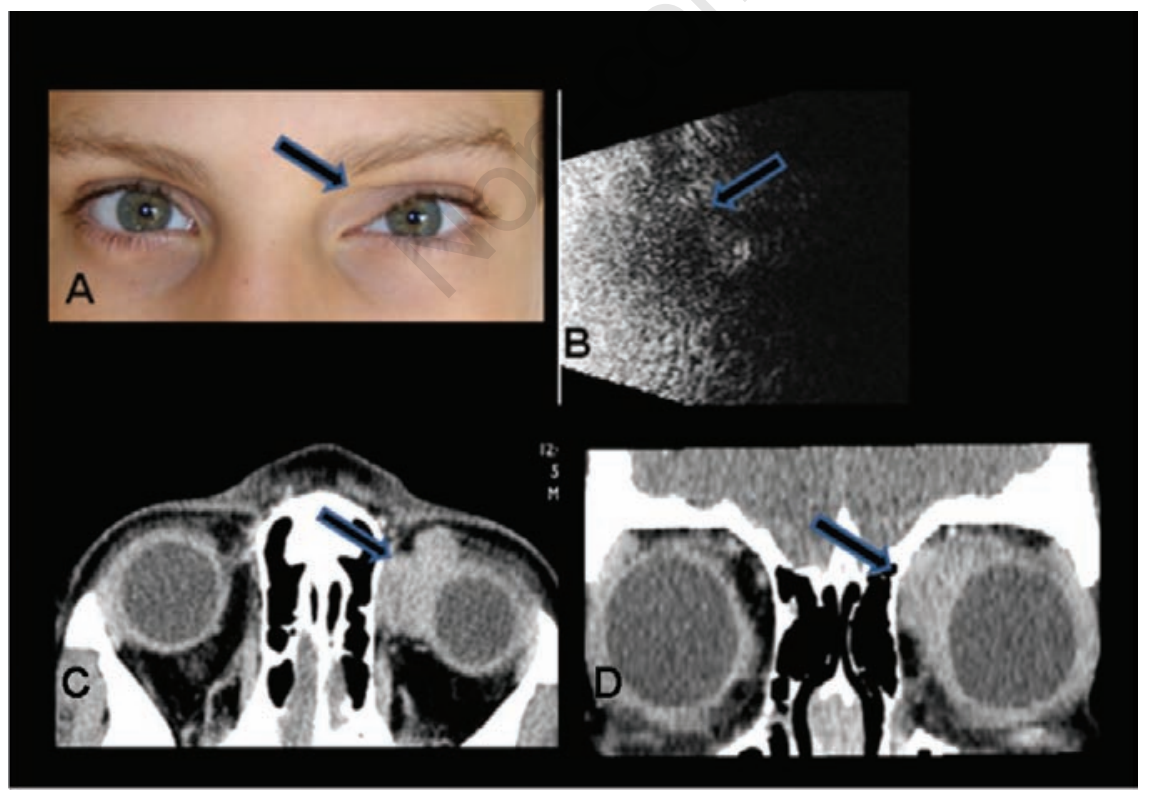

Figure 7. A) Left eyelid edema (arrow). B) B-scan ultrasonography: peribulbar section showing a poorly-defined extra-conic solid mass (arrow) located in the anterior upper segment of the right orbit-the eye is not visible. C) and D) CT scans showed a lobular non-encapsulated mass (arrow), featuring the same density as the extra-ocular muscles. indicated vascularized tissue, presenting with right medial canthal swelling. The patient was initially diagnosed with dacryocystitis and treated with oral antibiotics, followed by incision and drainage of a presumed lacrimal sac abscess. Rapid recurrence of the swelling led to further clinical evaluation, including a maxillofacial CT, which revealed an extensive nasal and orbital mass that was consistent with embryonal rhabdomyosarcoma on histopathologic analysis. ${ }^{7}$ Lazaridou et al. described a case of orbital rhabdomyosarcoma masquerading as a lacrimal mucocele in a newborn infant. $^{8}$ On the other hand, Cota et al. described an orbital abscess in a 6-year-old boy masquerading as a rhabdomyosarcoma ${ }^{9}$

In our case series, an expert sonographer and three different expert radiologists made an initially incorrect diagnosis in all cases. Usually, on ultrasonographical examinations, rhabdomyosarcoma appears as a solid homogeneous mass, with moderate reflectivity and mild sound attenuation. ${ }^{10}$ Sometimes septa can be seen separating different reflectivity structures. Blood flow is often detectable, and bone erosion is sometimes present.

On CT scans, rhabdomyosarcoma appears as a solid, defined, homogeneous formation, isodense to the skeletal muscles, with a regular internal structure, and moderate enhancement after contrast administration. In most cases the bone is spared, and only in more evolved forms is bone thinning or erosion present. Sometimes CT scan shows micro internal vaults. ${ }^{11}$ On T1-weighted MRI, rhabdomyosarcoma may appear hypointense with respect to the orbital fat. On proton density and T2weighted MRI, hypointensity, isointensity, and even hyperintensity may be appreciable with respect to both the extraocular muscles and the orbital fat. ${ }^{12}$

Imaging of lymphangioma often shows a multicystic mass with lobular margins. Inside the cyst an air-fluid level is often present. On MRI, lymphangioma shows hypointensity to the vitreous in T1 weighted images and hyperintensity in T2. The lymphangioma rim may be

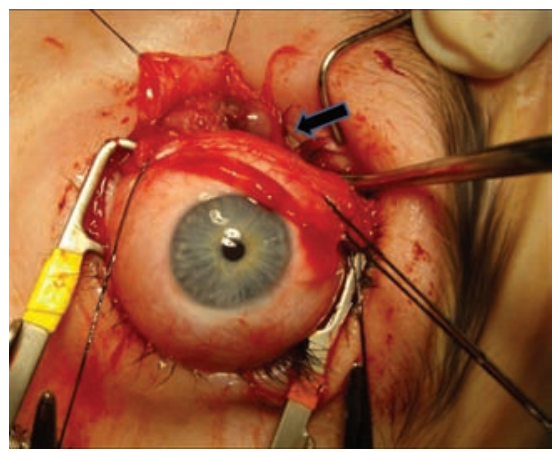

Figure 8. Excisional biopsy. 
Table 1. Comparison of suspected diagnosis and imaging with the final histological diagnosis, of cases reported in the literature and the cases described in this paper.

\begin{tabular}{|c|c|c|c|}
\hline Author & Suspected diagnosis & Imaging & Final diagnosis \\
\hline Seedat et $a l^{5}$ & $\begin{array}{l}\text { Suspected subperiosteal abscess in a } \\
\text { patient with acute sinusitis }\end{array}$ & $\begin{array}{l}\text { CT scan: ring-enhancing lesion within the orbit typical } \\
\text { of an orbital subperiosteal abscess. }\end{array}$ & Rhabdomyosarcoma \\
\hline Fetkenhour et al. ${ }^{6}$ & $\begin{array}{l}\text { Suspected lymphangioma in a patient with } \\
\text { proptosis of her right eye and painless } \\
\text { swelling of the right upper eyelid }\end{array}$ & $\begin{array}{l}\text { MRI: heterogeneous soft tissue mass with no bone } \\
\text { erosion and internal septa } \\
\text { MRI Tl- images: the central area of the mass was } \\
\text { hypointense, suggestive of proteinaceous material } \\
\text { and a peripheral hyperintense rim indicated } \\
\text { vascularized tissue }\end{array}$ & Rhabdomyosarcoma \\
\hline Burkat et al. ${ }^{7}$ & $\begin{array}{l}\text { Suspected dacryocystitis in a patient with } \\
\text { swelling in the right medial canthus }\end{array}$ & $\begin{array}{l}\text { MR-enhanced images: the central area of the mass } \\
\text { was hypointense, suggestive of proteinaceous material, } \\
\text { and a peripheral hyperintense rim indicated } \\
\text { vascularized tissue }\end{array}$ & Rhabdomyosarcoma \\
\hline Lazaridou et al. ${ }^{8}$ & $\begin{array}{l}\text { Suspected lacrimal mucocele in a newborn } \\
\text { patient with epiphora and swelling below } \\
\text { the left medial canthus }\end{array}$ & CT: homogenously hypodense mass & Rhabdomyosarcoma \\
\hline Our case \#1 & $\begin{array}{l}\text { Suspected chalazion or lymphangioma in } \\
\text { a patient with left inferior eyelid swelling }\end{array}$ & $\begin{array}{l}\text { CT: solid mass in the medial-inferior part of the orbit, } \\
\text { homogeneous and hyperdense after contrast } \\
\text { enhancement }\end{array}$ & Rhabdomyosarcoma \\
\hline Our case \#2 & $\begin{array}{l}\text { Suspected lymphangioma in a patient with } \\
\text { left eye exophthalmus }\end{array}$ & $\begin{array}{l}\text { CT: well-defined lesion with clear margins in the medial } \\
\text { segment of the left orbit, with several chambers, } \\
\text { which were more evident after contrast enhancement } \\
\text { MRI: irregular isointense tissue with an isointense } \\
\text { peripheral rim and some central areas with } \\
\text { a hyperintense signal }\end{array}$ & Rhabdomyosarcoma \\
\hline Our case \#3 & $\begin{array}{l}\text { Suspected lymphangioma in a patient with } \\
\text { superior left eyelid edema }\end{array}$ & $\begin{array}{l}\text { CT: lobular non-encapsulated mass, featuring the same } \\
\text { density as the extra-ocular muscles, well-defined against the } \\
\text { surrounding soft tissues, with diffuse contrast enhancement }\end{array}$ & Rhabdomyosarcoma \\
\hline
\end{tabular}

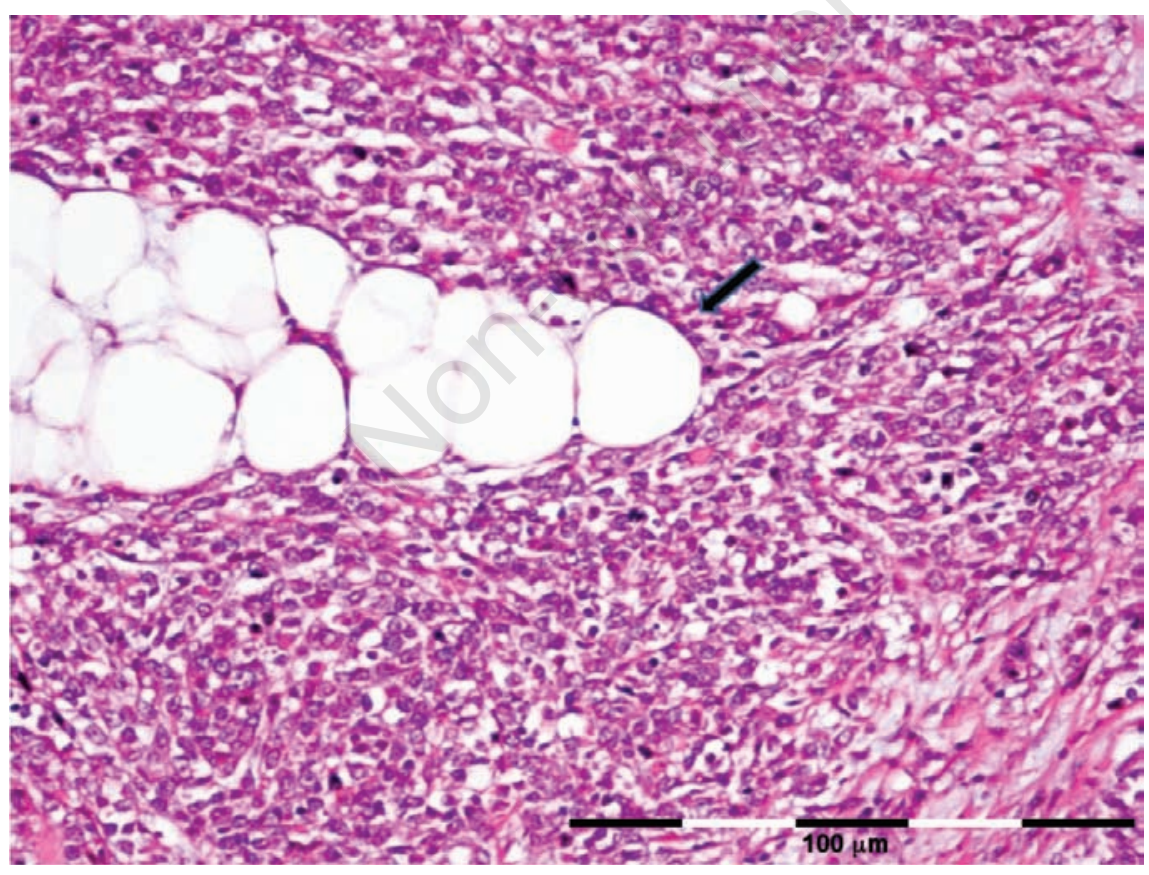

Figure 9. Histology (x100, with hematoxylin and eosin staining, scale bar: $100 \mu \mathrm{m}$ ) showed large and poorly differentiated cells infiltrating orbital fat (arrow). minimally enhanced. ${ }^{13}$

In all three of our cases, the lesion presented as an oval mass with clear margins, without any evidence of bone erosion. Orbital structures adjacent to the lesion were compressed and displaced but not infiltrated. Enhancement after contrast medium on CT in all cases showed a diffuse and patchy impregnation, more accentuated at the periphery of the lesion. In all lesions, imaging showed the presence of internal vaults that were particularly evident after the injection of contrast medium. These findings led to a diagnosis of lymphangioma, but this was not confirmed by histology.

Diagnostic imaging is useful to determine the location, size, and relationship with other orbital structures, as well as bone erosion and intracranial extension, but often ultrasonographic $^{13}$ and radiological ${ }^{4-10}$ characteristics of different pathologies overlap and thus do not allow a clear diagnosis. Imaging techniques are an important aid in the diagnosis of orbital pathologies, but are often inconclusive.

Management should be based first of all on clinical findings, and imaging findings should be treated with some care. The appropriate diagnosis of orbital rhabdomyosarcoma requires close cooperation and communication between the radiologist and the ophthalmologist. Rhabdomyosarcoma should be suspected 
whenever the clinical presentation of a rapidly progressive unilateral exophthalmos or eyelid swelling is observed in a child, and an exicional biopsy must always be considered. A prompt, correct diagnosis can be lifesaving.

\section{References}

1. Gurney JG, Young Jr JL, Roffers SD, et al. Soft tissue sarcomas. In: Ries LAG, Smith MA, Gurney JG, et al., eds. Cancer Incidence and Survival Among Children and Adolescents: United States SEER Program 1975-1995. Bethesda, MD: National Cancer Institute; 1999. National Institutes of Health publication NIH 99-4649.

2. Ducrey N, Nenadov-Beck M, Spahn B. Update of orbital rhabdomyosarcoma therapy in children. J Fr Ophthalmol 2002; 25:298-302.
3. Shields CL, Shields JA, Honaver SG, Demirci H. Clinical spectrum of primary ophthalmic rhabdomyosarcoma. Ophthalmology 2001;108: 2284-92.

4. Mishra A, Abuhajer R, Alsawidi K, et al. Congenital orbital lymphangioma in a 20 years old girl. A case report and review of literature. Libyan J Med 2009;4:162-3.

5. Seedat RY, Hamilton PD, de Jager LP. Orbital rhabdomyosarcoma presenting as an apparent orbital subperiosteal abscess. Int J Pediatr Otorhinolaryngol 2000;52: 177-81.

6. Frtkenhour DS, Shields CL, Chao AN, et al. Orbital cavitary Rhabdomyosarcoma masquerading as lymphangioma. Arch Ophthalmol 2001;119:1208-10.

7. Burkat CN, Lucarelli MJ. Rhabdomyo-sarcoma masquerading as acute dacryocystitis. Ophthal Plast Reconstr Surg 2005;21: 456-8.

8. Lazzaridou, Nabili S, Lavv T. Orbital rhab- domyosarcoma masquerading as a mucocele. J Pediatr Ophthalmol Strabismus 2008;45:306-8.

9. Cota N, Chandna A, Abernethy LJ. Orbital abscess masquerading as a rhabdomyosarcoma. J AAPOS 2000;4(5):318-20.

10. Sartor K, ed. MR Imaging of the Skull and Brain: A Correlative Text-Atlas. Berlin, New York, NY: Springer-Verlag; 1992.

11. Cooper S, Munk PL, Downey DB, et al. Findings of magnetic resonance and colour-flow Doppler imagining of orbital embryonal rhabdomyosarcoma. Can Assoc Radiol J 1994;45:217-220.

12. Karcioglu ZA, Hadjistilianou D, Rozans M, DeFrancesco S. Orbital rhabdomyosarcoma. Cancer Control 2004;11:328-33.

13. Neudorfer M, Leibovitch I, Stolovitch C, et al. Intraorbital and periorbital tumors in children-value of ultrasound and color Doppler imaging in the differential diagnosis. Am J Ophthalmol 2004;137:1065-72. 